\title{
Optimal timing of contrast-enhanced three-dimensional magnetic resonance left atrial angiography before pulmonary vein ablation
}

\author{
Susanne Löbe ${ }^{1}$, Claudia Leuthäusser ${ }^{1}$, Alexander Pölkow ${ }^{1}$, Sebastian Hilbert ${ }^{1}$, \\ Philipp Sommer ${ }^{1}$, Andreas Bollmann ${ }^{1}$, Gerhard Hindricks ${ }^{1,2}$, \\ Ingo Paetsch ${ }^{1}$, Cosima Jahnke ${ }^{1}$
}

${ }^{1}$ Department of Electrophysiology, HELIOS Heart Center Leipzig - University of Leipzig, Germany ${ }^{2}$ Leipzig Heart Institute, Leipzig, Germany

\begin{abstract}
Background: To achieve high image quality of cardiovascular magnetic resonance (CMR) pulmonary vein $(P V)$ angiography prior catheter ablation in patients with atrial fibrillation, optimal timing of the angiographic sequence during contrast agent passage is important. The present study identified influential cardiovascular parameters for prediction of contrast agent travel time.

Methods: One hundred six consecutive patients underwent a CMR examination including three-dimensional (3D) contrast-enhanced PV angiography with real-time bolus tracking prior to catheter ablation. Correct scan timing was characterized by relative signal enhancement measurements in the pulmonary artery, left atrium (LA), and ascending aorta. Furthermore, left- and right-ventricular function, left- and right-atrial dimensions, presence of mitral or tricuspid insufficiencies, and main pulmonary artery diameter were determined.

Results: The highest relative signal enhancement in LA demonstrated optimal scan timing. Contrast agent travel time showed wide variability (range: 12-42 s; mean: $18 \pm 4 \mathrm{~s}$ ). On univariate analysis, most cardiovascular parameters correlated with contrast agent travel time while on multivariate analysis left-and right-ventricular function remained the only independent predictors, but overall a poor fit to the data (adjusted $R^{2}, 27.5 \%$ ) was found.

Conclusions: Contrast agent travel time was mainly influenced by left-and right-ventricular function but prediction models poorly fitted the data. Thus, 3D PV angiography prior to PV ablation procedures necessitates real-time assessment, with visual determination of individual contrast agent passage time to ensure consistently high CMR image quality. (Cardiol J 2021; 28, 4: 558-565)

Key words: cardiovascular magnetic resonance imaging, angiography, pulmonary vein, atrial fibrillation, catheter ablation
\end{abstract}

\section{Introduction}

Atrial fibrillation (AF) is a common type of cardiac arrhythmia with a greater prevalence in the elderly [1] and in patients with cardiac comorbidities. Prior studies revealed the pulmonary veins (PVs) as important triggers for initializing and sustaining $\mathrm{AF}$ [2].
Treatment of AF with catheter ablation aimed at the electrical isolation of PVs is nowadays widely employed to prevent recurrent AF [3, 4].

Prior to PV isolation procedures, three-dimensional (3D) imaging of the left atrium (LA) and $\mathrm{PV}$ anatomy is recommended for pre-procedural planning and therapy guidance during catheter

Address for correspondence: Susanne Löbe, MD, Department of Electrophysiology, HELIOS Heart Center Leipzig — University of Leipzig, Struempellstr. 39, 04289 Leipzig, Germany, tel: +49 341865 1431, fax: +49 3418651460 , e-mail: Susanneloebe@hotmail.com

Received: 3.07.2019 Accepted: 3.11.2019

This article is available in open access under Creative Common Attribution-Non-Commercial-No Derivatives 4.0 International (CC BY-NC-ND 4.0) license, allowing to download articles and share them with others as long as they credit the authors and the publisher, but without permission to change them in any way or use them commercially. 
ablation. These high-resolution images allow for accurate assessment of anatomical variants and can be obtained by either multi-detector computed tomography (MDCT) or cardiovascular magnetic resonance (CMR) [4, 5]. However, MDCT exposes the patient to iodinated contrast agents and ionizing radiation [6] and AF is a chronic, progressive disease making repeat examinations during life-time very likely. CMR imaging has the additional benefit of combining PV angiography with functional cardiac imaging or myocardial tissue characterization (e.g. LA fibrosis) during a single-session examination [7]. Consequently, CMR may be considered the preferred imaging approach; the segmented 3D CMR mesh models of the LA and the PVs can be easily co-registered in the electroanatomical mapping systems and subsequently used for catheter guidance during the ablation procedures, thereby significantly reducing overall radiation exposure time $[8,9]$.

High spatial resolution together with high image quality of contrast-enhanced PV angiography is mandatory for electrophysiological procedures and hence, the optimal scan timing during contrast agent passage plays a pivotal role. Whether the presence of $\mathrm{AF}$ during CMR imaging affects the contrast agent travel time has not yet been investigated. Moreover, predicting the optimal scan timing in an individual patient prior to CMR angiography would be highly desirable. Consequently, the present study sought to evaluate various routine cardiovascular parameters with regard to their influence on contrast agent bolus travel time

\section{Methods}

\section{Patients}

One hundred six consecutive patients $(62 \pm 10$ years, 61 men) with AF or non-isthmus dependent left-atrial flutter underwent CMR imaging prior to clinically indicated catheter ablation. Patients with known contraindications to CMR imaging were not considered. Detailed patient characteristics are provided in Table 1 . The study was conducted in accordance with the local institutional review board and the standards of the University of Leipzig ethics committee. Written informed consent was obtained from all patients.

\section{CMR imaging protocol}

All CMR examinations were performed using a 1.5T MR scanner system (Philips Ingenia, Best, The Netherlands) equipped with Omega HP gradients $(45 \mathrm{mT} / \mathrm{m}, 200 \mathrm{~T} / \mathrm{m} / \mathrm{s})$ and a 28 -element
Table 1. Patient characteristics $(n=106)$.

\begin{tabular}{lc}
\hline Age [years] & $62 \pm 10$ \\
Women & $45(43 \%)$ \\
Sinus rhythm during CMR-study & $55(52 \%)$ \\
Heart rate during CMR-study [1/min] & $78 \pm 26$ \\
Pulmonary disease & $6(6 \%)$ \\
Body mass index [kg/m ${ }^{2}$ ] & $29 \pm 5$ \\
Left ventricular ejection fraction [\%] & $53 \pm 11$ \\
Left ventricular end-diastolic & $157 \pm 54$ \\
volume [mL] & \\
Cardiac output [L/min] & $5.9 \pm 1.6$ \\
Right ventricular ejection fraction [\%] & $42 \pm 8$ \\
Left atrial area [cm ${ }^{2}$ ] & $29 \pm 8$ \\
Right atrial area [cm ${ }^{2}$ ] & $24 \pm 6$ \\
Pulmonary artery diameter [mm] & $26 \pm 4$ \\
Systolic pulmonary artery & $27 \pm 8$ \\
pressure [mmHg] & $10(9 \%)$ \\
Mitral regurgitation $\geq$ grade 2 & $8(8 \%)$ \\
Tricuspid regurgitation $\geq$ grade 2 & \\
\hline
\end{tabular}

Data are provided as mean \pm standard deviation or number (percent). CMR - cardiovascular magnetic resonance

array coil with full in-coil signal digitalization combined with optical transmission. Conventional cine imaging was performed in all cardiac standard geometries (short axis geometries and long axis geometries, i.e. 4-, 3-, and 2-chamber orientation) using steady-state free precession (SSFP) sequences during end-expiratory breathholds with a prospective electrocardiogram (ECG)-gating acquisition. In addition, a 3D navigator-gated, balanced turbo field echo (bTFE) sequence was acquired in transversal slice orientation with full coverage of the great thoracic vessels. Furthermore, phase-contrast flow measurements were performed for the assessment of the cardiac output. Finally, contrast-enhanced 3D CMR angiography of the LA and PVs was performed during inspiratory breath-holding using a non-ECG triggered spoiled gradient echo sequence (TR/TE/flip angle: $2.2 \mathrm{~ms} / 0.8 \mathrm{~ms} / 30^{\circ}$, isotropic spatial resolution: $1.0 \times 1.0 \times 1.0 \mathrm{~mm}^{3}$ ). During intravenous bolus injection of $0.1 \mathrm{mmol} / \mathrm{kg}$ Gad-DTPA (Magnograf ${ }^{\circledR}$, injection rate $4.0 \mathrm{~mL} / \mathrm{s}$ ) followed by a $25 \mathrm{~mL}$ saline flush at the same injection rate, integrated realtime bolus tracking in coronal slice orientation (slice thickness, $150 \mathrm{~mm}$; in-plane spatial resolution, $1.7 \times 1.7 \mathrm{~mm}^{2}$; temporal resolution, $680 \mathrm{~ms}$ ) allowed for visual determination of the sequence start as performed by a trained CMR operator; the angiographic scan was initiated when the contrast agent bolus arrived in the left atrium. 


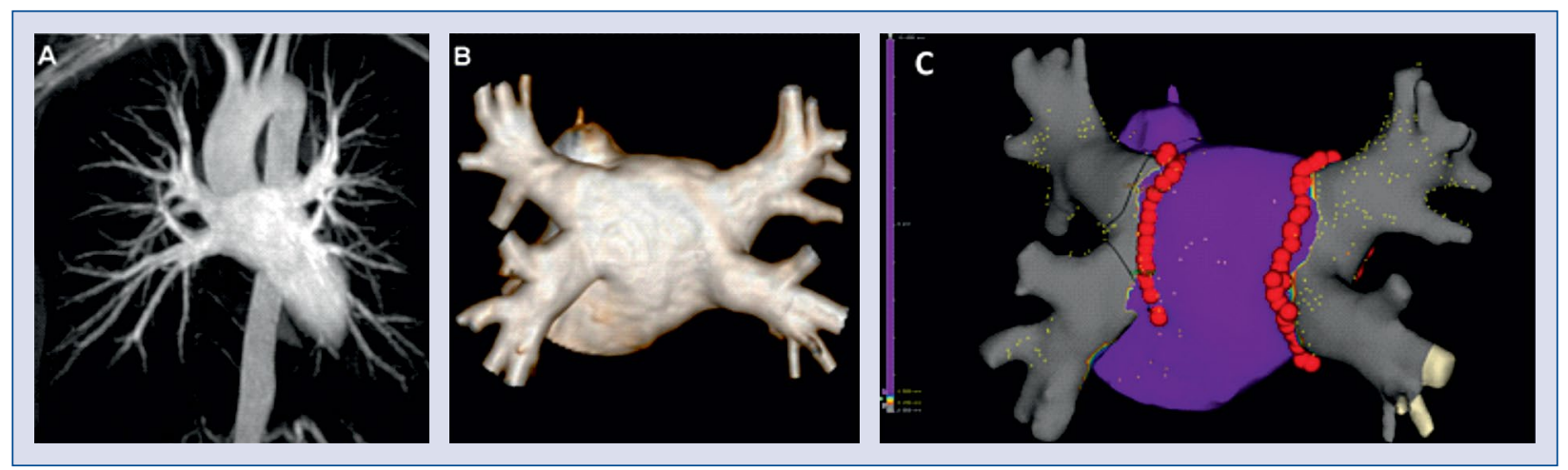

Figure 1. A. Maximum-intensity projection of three-dimensional (3D) contrast-enhanced cardiovascular magnetic resonance (CMR) angiography of the left atrium and pulmonary veins; accurate timing led to the highest relative signal enhancement in left atrium/pulmonary veins; B. Segmented volume rendering reconstruction of the left atrium and pulmonary veins; subsequently generated 3D CMR mesh model can be easily integrated into electroanatomical mapping systems for guidance of catheter ablation procedures; C. Image fusion of electroanatomical map (EnSite Precision, St. Jude Medical, St. Paul, MN, US) and CMR mesh model of left atrium and pulmonary veins during electrophysiological ablation procedure.

\section{CMR image analysis}

Cine imaging was used to determine leftventricular volumes and function, right-ventricular function and left-/right-atrial size according to standard definitions. Maximal diameter of the main pulmonary artery (PA) was measured on 3D bTFE scan.

In order to objectively determine the correct scan timing of PV angiography, signal intensity measurements were carried out in the PA, the LA, the ascending and descending aorta, and in the adipose tissue of the anterior chest wall; relative signal enhancement was calculated by dividing the maximum signal intensity of the target region by the signal intensity of the reference tissue (= subcutaneous fat).

\section{Echocardiography}

In all patients, two-dimensional transthoracic echocardiography was performed within 1 week prior the CMR examination using a commercially available ultrasound system (Vivid 7, General Electric, Milwaukee, WI, USA) equipped with a $3.5 \mathrm{MHz}$ transducer. Recordings were made in parasternal long- and short-axis, as well as apical 4- and 2-chamber views. Valve morphology and function were assessed according to the guidelines of the European Society of Cardiovascular Imaging [10] and the American Society of Echocardiography [11]: the severity of mitral and tricuspid regurgitation was graded on a four-point scale. In addition, systolic pulmonary artery pressure (PAP) was estimated based on tricuspid regurgitation velocity.

\section{Statistical analysis}

Continuous variables are stated as mean \pm standard deviation if normally distributed. Numbers and ratios were used to describe categorical variables. The Kolmogorov-Smirnov test was used to assess normal distribution. The $\chi^{2}$ test was used for comparisons between groups in case of categorical variables; the Student t-test was applied for continuous variables. To determine the relationship of contrast agent travel time and cardiovascular parameters, univariate logistic regression analysis was done. Parameters which yielded as statistically significant in univariate logistic regression analyses were assessed by multivariate logistic regression analysis. In addition, univariate and multivariate regression analysis was performed in the subgroups of patients presenting with sinus rhythm or AF during CMR examination and estimation models based on polynomial data fitting were derived. A two-tailed p-value $<0.05$ was considered significant.

\section{Results}

\section{Patient characteristics}

Cardiovascular magnetic resonance examinations were successfully completed in all 106 patients (Fig. 1). Patient demographics are summarized in Table 1. 


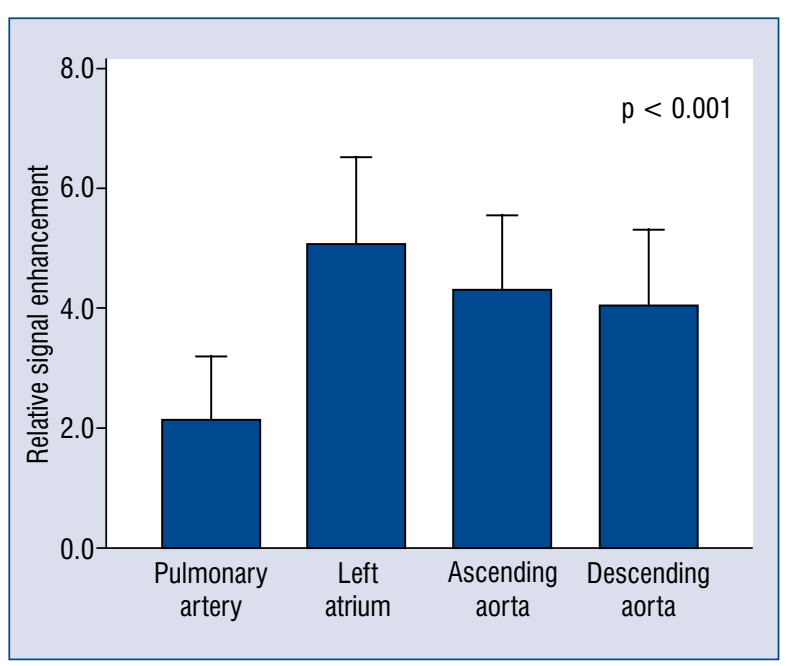

Figure 2. Measurements of relative signal enhancement (mean \pm standard deviation) confirmed the highest values in left atrium $(5.1 \pm 1.5 ; p<0.001)$ in comparison to the pulmonary artery $(2.1 \pm 1.1)$, ascending $(4.3 \pm 1.2)$ and descending aorta $(4.0 \pm 1.3)$, respectively.

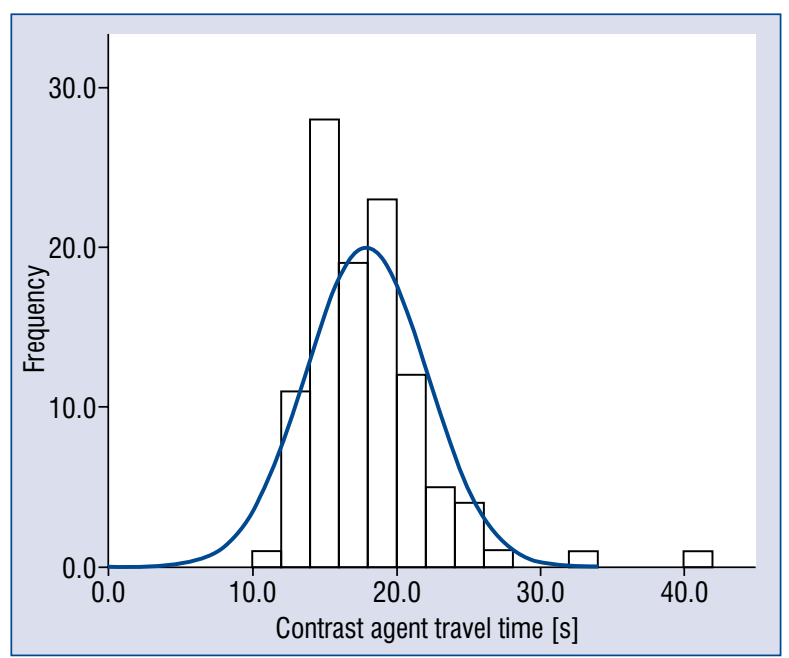

Figure 3. Histogram plot of contrast agent travel time in all patients demonstrated a wide variability (range 12-42 s; mean $18 \pm 4 \mathrm{~s}$ ); the blue line indicates fitted normal distribution.

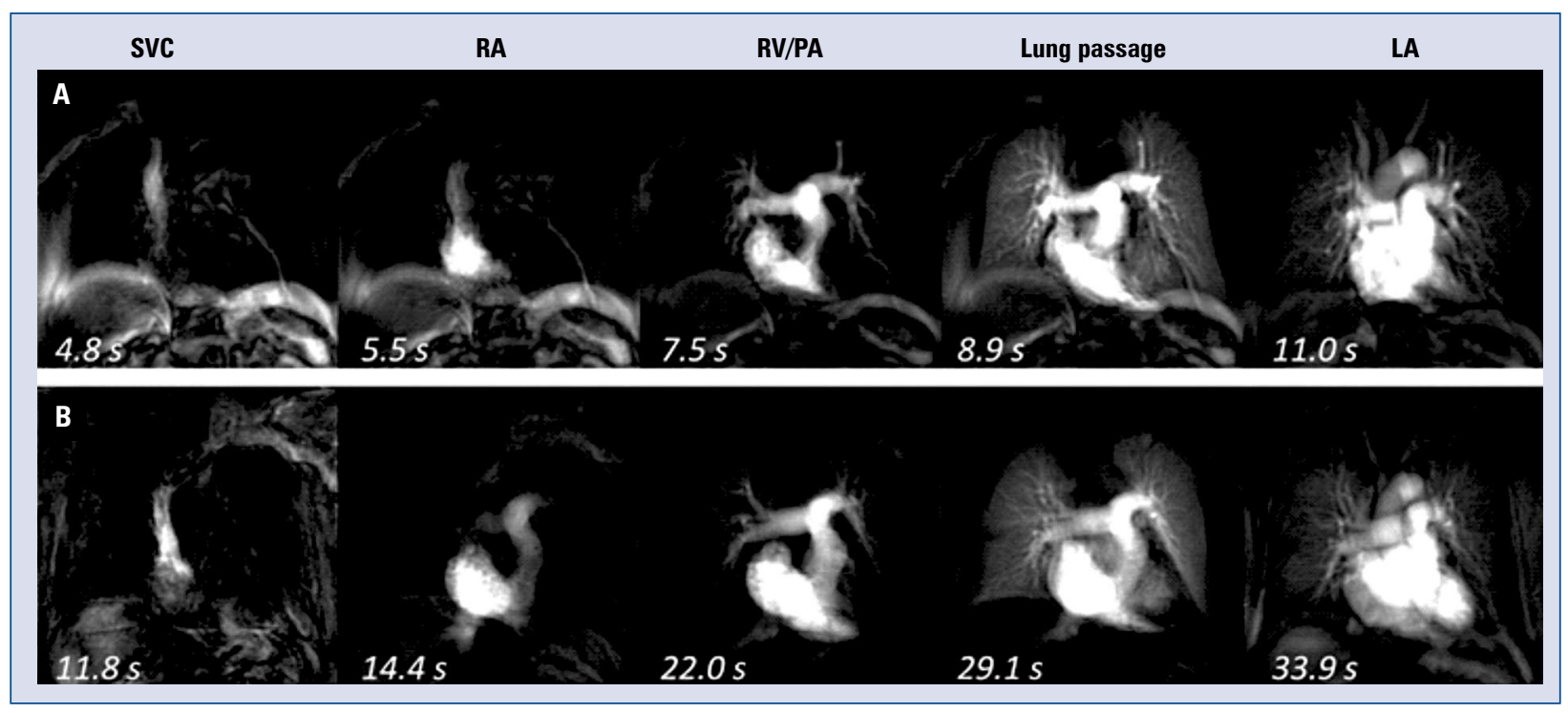

Figure 4. Real-time cardiovascular magnetic resonance (CMR) bolus tracking during contrast agent passage: representative extracted frames of contrast agent bolus passage are shown demonstrating its arrival in superior vena cava $(\mathrm{SVC})$, right atrium (RA), right ventricle (RV)/pulmonary artery (PA), lung and left atrium (LA); subsequently, the three-dimensional angiographic imaging sequence was started; A. Sinus rhythm (heart rate 78/min), LV-EF 64\%, RV-EF $61 \%$, LA $18 \mathrm{~cm}^{2}$, RA $17 \mathrm{~cm}^{2}$, MI grade 1; B. Atrial fibrillation (heart rate approx. 100/min), LV-EF 23\%, RV-EF 24\%, LA $29 \mathrm{~cm}^{2}$, RA $27 \mathrm{~cm}^{2}$, MI grade 1; LV — left ventricular; EF — ejection fraction.

\section{Contrast agent travel time}

The relative signal enhancement of large thoracic vessels and cardiac cavities served as a quality measure of accurate timing of the 3D angiographic scan with the highest relative signal enhancement observed in the LA (LA $5.1 \pm 1.5$; PA $2.1 \pm 1.1$; ascending aorta $4.3 \pm 1.2$; descending aorta $4.0 \pm 1.3 ; \mathrm{p}<0.001$; Fig. 2 ); in $100 \%$
(106/106) and 98\% (104/106) of patients relative signal enhancement was found to be higher in the LA when compared to the main PA and the ascending aorta, respectively. The travel time of the contrast agent bolus was normally distributed (Kolmogorov-Smirnov test, $\mathrm{p}=0.11$ ) and demonstrated a wide variability (range $12-42 \mathrm{~s}$; mean $18 \pm 4 \mathrm{~s}$; median $17 \mathrm{~s}$; Figs. 3, 4). 
Table 2. Univariate and multiple linear regression (MLR) analysis ( $p$-values) for the prediction of the real-time tracking time.

\begin{tabular}{|c|c|c|c|c|c|c|}
\hline & \multicolumn{2}{|c|}{ All patients } & \multicolumn{2}{|c|}{$\begin{array}{c}\text { SR during CMR } \\
\text { study }\end{array}$} & \multicolumn{2}{|c|}{$\begin{array}{c}\text { AF during CMR } \\
\text { study }\end{array}$} \\
\hline & Univariate & MLR & Univariate & MLR & Univariate & MLR \\
\hline Age & 0.02 & 0.14 & 0.07 & & 0.79 & \\
\hline Sex & 0.06 & & 0.1 & & 0.22 & \\
\hline SR during CMR-study & 0.01 & 0.95 & & & & \\
\hline Heart rate during CMR-study & 0.12 & & 0.63 & & 0.92 & \\
\hline Body mass index & 0.36 & & 0.49 & & 0.87 & \\
\hline LVEF & $<0.001$ & 0.01 & $<0.001$ & 0.001 & 0.03 & 0.16 \\
\hline LVEDV & 0.004 & 0.17 & $<0.001$ & 0.51 & 0.20 & \\
\hline Cardiac output & 0.23 & & 0.21 & & 0.46 & \\
\hline RVEF & $<0.001$ & $<0.001$ & $<0.001$ & 0.002 & 0.013 & 0.057 \\
\hline LA & 0.001 & 0.65 & 0.046 & 0.34 & 0.052 & \\
\hline RA & 0.001 & 0.61 & 0.003 & 0.30 & 0.15 & \\
\hline Pulmonary artery diameter & 0.02 & 0.24 & 0.069 & & 0.28 & \\
\hline Systolic PAP & 0.025 & 0.73 & 0.035 & 0.56 & 0.57 & \\
\hline Mitral regurgitation $\geq$ grade 2 & 0.001 & 0.27 & 0.008 & 0.52 & 0.17 & \\
\hline Tricuspid regurgitation $\geq$ grade 2 & 0.01 & 0.58 & 0.03 & 0.85 & 0.39 & \\
\hline
\end{tabular}

CMR - cardiovascular magnetic resonance; SR - sinus rhythm; AF — atrial fibrillation; LVEF — left ventricular ejection fraction; LVEDV left ventricular end-diastolic volume; RVEF — right ventricular ejection fraction; LA — left atrial dimension; RA — right atrial dimension;

PAP — pulmonary arterial pressure

In order to simulate angiographic scan timing done without real-time display of contrast agent bolus passage, derived mean and median values with an allowed deviation of \pm 1 s were employed as "fixed" timing parameters to the current study population: theoretically, such an approach would have yielded successful timing in only $16 \%$ and $20 \%$ of patients within the predefined ranges of $17-19 \mathrm{~s}$ and $16-18 \mathrm{~s}$, respectively.

To determine the influence of various routine cardiovascular parameters on the contrast agent travel time, univariate analysis was performed and revealed a significant correlation of the contrast agent travel time with age, heart rhythm, left and right ventricular ejection fraction (LVEF, RVEF), left ventricular end-diastolic volume, left and right atrial size, PA diameter, presence of mitral or tricuspid regurgitation and systolic PAP, respectively (Table 2). On multivariate analysis, LVEF and RVEF remained the only independent predictors of the contrast agent travel time $(\mathrm{p}=0.002$ and $\mathrm{p}<0.001$, respectively); however, the adjusted $\mathrm{R}^{2}$ of $27.5 \%$ indicated that the regression model poorly fitted the data.

In addition, subgroup analysis in patients presenting with sinus rhythm or AF during the CMR examination was carried out (Table 2) using linear regression analysis: LVEF and RVEF remained the only independent predictors of contrast agent travel time in sinus rhythm patients while in $\mathrm{AF}$ patients none of the cardiovascular parameters reached the level of significance (adjusted $\mathrm{R}^{2}$-values, $54 \%$ and $12 \%$, respectively).

In order to derive estimation models for the prediction of contrast agent travel time in patients with sinus rhythm, LVEF and RVEF were employed in polynomial curve fitting procedures. Based on $\mathrm{R}^{2}$ change, quadratic models were identified to represent a favorable compromise between model complexity and routine applicability (Table 3 ). Though significant, explaining only an additional $2 \%$ or $9 \%$ of the variance was considered not to justify rendering the model even more complex and hence, the cubic fit was rejected. Respective estimation models for the calculation of predicted contrast agent travel time are provided in Table 4 .

\section{Discussion}

The present study evaluated the influence of various cardiovascular parameters on the contrast agent travel time as assessed during contrastenhanced CMR PV angiography prior to catheter ablation of AF. The main findings were as follows: 
Table 3. Polynomial regression analysis to determine the influence of left and right ventricular ejection fraction (LVEF, RVEF) on contrast agent travel time in patients with sinus rhythm ( $n=55)$.

\begin{tabular}{lccccccc}
\hline & \multicolumn{3}{c}{ LVEF } & & \multicolumn{3}{c}{ RVEF } \\
\cline { 2 - 3 } & $\mathbf{R}^{2}$ & Adjusted $\mathbf{R}^{2}$ & $\mathbf{R}^{2}$ change & & $\mathbf{R}^{2}$ & Adjusted $\mathbf{R}^{2}$ & $\mathbf{R}^{2}$ change \\
\hline Linear model & 0.43 & 0.42 & 0.43 & & 0.33 & 0.32 & 0.33 \\
Quadratic model & 0.56 & 0.54 & 0.13 & & 0.55 & 0.54 & 0.22 \\
Cubic model & 0.58 & 0.55 & 0.02 & & 0.64 & 0.63 & 0.09 \\
\hline
\end{tabular}

Table 4. Estimation models for calculation of contrast agent travel time (given in seconds) in patients with sinus rhythm.

\begin{tabular}{|c|c|}
\hline \multicolumn{2}{|c|}{ LVEF-based models } \\
\hline Linear & Contrast agent travel time $[\mathrm{s}]=35-0.33 \times \mathrm{LVEF}$ \\
\hline Quadratic & Contrast agent travel time $[\mathrm{s}]=58-1.4 \times \mathrm{LVEF}+0.01 \times \mathrm{LVEF}^{2}$ \\
\hline \multicolumn{2}{|c|}{ RVEF-based models } \\
\hline Linear & Contrast agent travel time $[\mathrm{s}]=31-0.33 \times \mathrm{RVEF}$ \\
\hline Quadratic & Contrast agent travel time $[\mathrm{s}]=61-1.8 \times \mathrm{RVEF}+0.02 \times \mathrm{RVEF}^{2}$ \\
\hline
\end{tabular}

LVEF — left ventricular ejection fraction (in \%); RVEF — right ventricular ejection fraction (in \%)

(1) on univariate analysis numerous cardiovascular parameters had an influence on contrast agent travel time, however (2) on multivariate analysis the only independent predictors were identified as LVEF and RVEF; (3) a reliable prediction of the contrast agent travel time was not accurately possible for every individual patient in particular in patients with AF during CMR examination; (4) in the subgroup of patients presenting with sinus rhythm contrast agent travel time may be determined from LVEF and RVEF using the proposed polynomial estimation model; (5) finally, visual determination of contrast agent bolus arrival in the target region (i.e. the LA) using a real-time tracking sequence enabled accurate timing of image data acquisition in all patients.

Three-dimensional angiographic determination of PV and left atrial anatomy can assist in preprocedural decision making (e.g. cryoablation vs. radiofrequency ablation technique) and is particularly important for anatomical guidance during the ablation procedure [4]. Depending on the operators' preferences, image fusion of pre-procedural CT/ /CMR anatomic 3D reconstructions with electroanatomical maps can contribute in facilitating complex AF ablation procedures [12]. In addition, high-resolution, 3D depiction of the LA and PV morphology resulting from image fusion reportedly increased the safety of the $\mathrm{AF}$ ablation procedures [13] and is fundamental in the prevention of rare, but severe procedure-related complications such as PV stenosis [14].

For high-quality, contrast-enhanced 3D CMR angiography accurate timing of bolus arrival in the LA/PV target region is of the essence. Although real-time tracking which permits the direct visualization of the bolus passage has been established for several years, data from a multicenter trial revealed a high proportion of technical failures due to timing errors of up to $25 \%$ for CMR angiography of the pulmonary arteries with a proportion of technically inadequate images ranging from $11 \%$ to $52 \%$ between different centers [15]. Obviously, a reliable prediction of the correct timing to assist the CMR operator would be highly desirable. In the current study population, the distribution of the contrast agent travel time showed a high variability which on theoretical simulation using a fixed timing value (mean or median) would have resulted in $84 \%$ or $80 \%$ of inaccurately timed angiographic scans, respectively, and thus, leading to inadequate contrast enhancement and impaired image quality in a majority of patients.

Consequently, the current study examined a variety of readily available cardiovascular parameters and tested their influence on the contrast agent travel time with the aim to better predict scan timing. While on univariate analysis several cardiovascular parameters were associated significantly with contrast agent travel time, left and right 
ventricular function remained the only independent predictors on multivariate analysis. However, the low adjusted $\mathrm{R}^{2}$ indicated that the regression model poorly fitted the data. A subgroup analysis of patients presenting with AF during CMR examination demonstrated that prediction of scan timing will almost invariably fail. On the other hand, the subgroup of sinus rhythm patients yielded a considerably higher adjusted $\mathrm{R}^{2}$ value suggesting that a prediction of the contrast agent travel time may be possible. An estimation model, using a linear fit provided an easily applicable approach in clinical routine by calculating 35 or 31 minus one-third of LVEF or RVEF, respectively. An improved, though more complex estimate could be achieved by applying a quadratic model given in Table 4 . However, the adjusted $\mathrm{R}^{2}$ values indicated that only $54 \%$ of the overall variation could be explained by the independent variables LVEF and RVEF. Thus, it must be noted that the possibilities to predict the contrast agent travel time in an individual patient are severely limited.

To overcome these timing challenges, alternative imaging approaches have been introduced. Free-breathing ECG-gated 3D SSFP sequences render the correct timing process needless: in a small patient study [16] a non-contrast enhanced imaging approach proved to be highly accurate with regard to PV diameter measurements when compared to contrast-enhanced CMR angiography. Another study [17] applied an accelerated free-breathing, ECG-triggered contrast-enhanced PV CMR angiographic scan with isotropic spatial resolution using compressed sensing, resulting in even further improvement of vessel sharpness when compared to conventional CMR angiography. However, the fundamental prerequisite for all these ECG-triggered imaging approaches consists in the presence of a regular sinus rhythm. But considering the patient population scheduled for PV angiography prior to electrophysiological ablation procedures, a high proportion of patients will present with $\mathrm{AF}$ and, thus, a high heart rate variability. In the present study, nearly half of the patients had $\mathrm{AF}$ during the CMR examination (48\%) and consequently, a non-ECG triggered imaging approach such as the conventional contrast-enhanced PV angiography is generally preferred.

A widely used alternative to real-time tracking of the contrast agent bolus is the administration of a small test bolus in order to estimate the arrival time in the target region. General disadvantages of test bolus timing include increased examination duration and background contamination by gadolinium (potentially leading to unfavorable pulmonary tissue enhancement and decreased PV conspicuity). More importantly, the arrival times for a small contrast agent dose and the full dose are not necessarily consistent. Finally, taking into account the high heart rate variability of $\mathrm{AF}$ patients with concomitant rapid changes of hemodynamics, it is evident that the test bolus strategy can be regarded inherently flawed in this particular patient population.

Finally, another important CMR imaging approach has become available with the advent of time-resolved 3D-CMR angiographic scans (so called " $4 \mathrm{D}-\mathrm{CMR}$ angiography" with time representing the fourth dimension). This scan technique allows the acquisition of full 3D-angiographic datasets of the thorax/large thoracic vessels in a time-resolved manner (i.e. usually every 4 to $6 \mathrm{~s}$ ) but this is at the expense of spatial resolution. The technique mostly obviates the need for accurate bolus timing since during post-processing a CMR expert selects the single, high signal enhancement 3D-dataset of the LA/the PVs for diagnostic evaluation and volume rendering/ /mesh reconstruction. However, the lower spatial resolution of $4 \mathrm{D}$-angiographic scans (non-isotropic datasets, typically $2.5 \times 2.5 \mathrm{~mm}^{2}$ in-plane resolution with $5 \mathrm{~mm}$ slice thickness) in comparison to bolus-tracking directed 3D-angiographic scans (preserved high isotropic spatial resolution, usually in the range of $1.0 \times 1.0 \mathrm{~mm}^{2}$ in-plane resolution with $1.0 \mathrm{~mm}$ slice thickness) should be taken into account when establishing a routine institutional angiographic protocol: with $4 \mathrm{D}-\mathrm{CMR}$ angiography small caliper PV anatomical variants (early small caliper branching of PV main ostia or accessory $\mathrm{PVs}$ e.g. right middle PVs, isolated roof top veins etc.) may be poorly visible or even missed and, if electrically active, may represent a possible focus for re-occurrence of AF. Hence, at our institution the interventional electrophysiologists generally prefer bolus-tracking directed, high spatial resolution 3D-angiography for anatomical procedural guidance.

\section{Conclusions}

For the determination of contrast agent travel time, left and right ventricular function were identified as the only independent predictors but regression models poorly fitted the data, particularly in patients with $\mathrm{AF}$ during CMR examination. Thus, 3D, PV angiography prior to PV ablation procedures necessitates real-time assessment 
with visual determination of individual contrast agent passage time to ensure consistently high CMR image quality.

\section{Conflict of interest: None declared}

\section{References}

1. Haim M, Hoshen M, Reges O, et al. Prospective national study of the prevalence, incidence, management and outcome of a large contemporary cohort of patients with incident non-valvular atrial fibrillation. J Am Heart Assoc. 2015; 4(1): e001486, doi: 10.1161/ JAHA.114.001486, indexed in Pubmed: 25609415.

2. Haïssaguerre M, Jaïs $P$, Shah DC, et al. Spontaneous initiation of atrial fibrillation by ectopic beats originating in the pulmonary veins. N Engl J Med. 1998; 339(10): 659-666, doi: 10.1056/ NEJM199809033391003, indexed in Pubmed: 9725923.

3. Kirchhof P, Benussi S, Kotecha D, et al. 2016 ESC Guidelines for the management of atrial fibrillation developed in collaboration with EACTS. Europace. 2016; 18(11): 1609-1678, doi: 10.1093/ europace/euw295.

4. Mansour M, Refaat M, Heist EK, et al. Three-dimensional anatomy of the left atrium by magnetic resonance angiography: implications for catheter ablation for atrial fibrillation. J Cardiovasc Electrophysiol. 2006; 17(7): 719-723, doi: 10.1111/j.15408167.2006.00491.x, indexed in Pubmed: 16836666.

5. Löbe S, Hilbert S, Dinov B, et al. High electrical activity at the connection site of previously undetected anomalous pulmonary venous drainage in a patient presenting with atrial fibrillation. Int J Cardiovasc Imaging. 2017; 33(11): 1845-1846, doi: 10.1007/ s10554-017-1184-1, indexed in Pubmed: 28589483.

6. Vyas HV, Greenberg SB, Krishnamurthy R. MR imaging and CT evaluation of congenital pulmonary vein abnormalities in neonates and infants. Radiographics. 2012; 32(1): 87-98, doi: 10.1148/rg.321105764, indexed in Pubmed: 22236895.

7. Marrouche NF, Wilber D, Hindricks G, et al. Association of atrial tissue fibrosis identified by delayed enhancement MRI and atrial fibrillation catheter ablation: the DECAAF study. JAMA. 2014; 311(5): 498-506, doi: 10.1001/jama.2014.3, indexed in Pubmed: 24496537.

8. Tops LF, Bax JJ, Zeppenfeld K, et al. Fusion of multislice computed tomography imaging with three-dimensional electroanatomic mapping to guide radiofrequency catheter ablation procedures. Heart Rhythm. 2005; 2(10): 1076-1081, doi: 10.1016/j. hrthm.2005.07.019, indexed in Pubmed: 16188585.
9. Dong J, Dickfeld T, Dalal D, et al. Initial experience in the use of integrated electroanatomic mapping with three-dimensional MR/ /CT images to guide catheter ablation of atrial fibrillation. J Cardiovasc Electrophysiol. 2006; 17(5): 459-466, doi: 10.1111/j.15408167.2006.00425.x, indexed in Pubmed: 16684014.

10. Lancellotti P, Tribouilloy C, Hagendorff A, et al. Recommendations for the echocardiographic assessment of native valvular regurgitation: an executive summary from the European Association of Cardiovascular Imaging. Eur Heart J Cardiovasc Imaging. 2013; 14(7): 611-644, doi: 10.1093/ehjci/jet105, indexed in Pubmed: 23733442.

11. Rudski LG, Lai WW, Afilalo J, et al. Guidelines for the echocardiographic assessment of the right heart in adults: a report from the American Society of Echocardiography endorsed by the European Association of Echocardiography, a registered branch of the European Society of Cardiology, and the Canadian Society of Echocardiography. J Am Soc Echocardiogr. 2010; 23(7): 685-713; quiz 786, doi: 10.1016/j.echo.2010.05.010, indexed in Pubmed: 20620859.

12. Kistler PM, Rajappan K, Harris S, et al. The impact of image integration on catheter ablation of atrial fibrillation using electroanatomic mapping: a prospective randomized study. Eur Heart J. 2008; 29(24): 3029-3036, doi: 10.1093/eurheartj/ehn453, indexed in Pubmed: 18931059.

13. Kettering K, Greil GF, Fenchel M, et al. Catheter ablation of atrial fibrillation using the Navx-/Ensite-system and a CT-/MRI-guided approach. Clin Res Cardiol. 2009; 98(5): 285-296, doi: 10.1007/s00392-009-0001-9, indexed in Pubmed: 19283334.

14. Hilbert S, Paetsch I, Bollmann A, et al. Pulmonary vein collateral formation as a long-term result of post-interventional pulmonary vein stenosis. Eur Heart J. 2016; 37(31): 2474, doi: 10.1093/ eurheartj/ehv753, indexed in Pubmed: 26795444.

15. Stein P, Chenevert TL, Fowler SE. Gadolinium-Enhanced magnetic resonance angiography for pulmonary embolism. Ann Intern Med. 2010; 152(7): 434-443, doi: 10.7326/0003-4819-1527-201004060-00008.

16. François CJ, Tuite D, Deshpande V, et al. Pulmonary vein imaging with unenhanced three-dimensional balanced steady-state free precession MR angiography: initial clinical evaluation. Radiology. 2009; 250(3): 932-939, doi: 10.1148/radiol.2502072137, indexed in Pubmed: 19164696.

17. Roujol S, Foppa M, Basha TA, et al. Accelerated free breathing ECG triggered contrast enhanced pulmonary vein magnetic resonance angiography using compressed sensing. J Cardiovasc Magn Reson. 2014; 16: 91, doi: 10.1186/s12968-014-0091-z, indexed in Pubmed: 25416082. 